Société d'histoire de la révolution de 1848 et des

révolutions du XIXe siècle

$63 \mid 2021$

Puissances de la Commune

\title{
Samuel BAMFORD, La Vie d'un radical anglais au temps de Peterloo
}

Traduction par Laurent Bury, édité par Fabrice Bensimon, avec le concours de Robert Poole, Paris, Éditions sociales, 2019

François Jarrige

\section{(2) OpenEdition}

\section{Édition électronique}

URL : https://journals.openedition.org/rh19/7970

DOI : $10.4000 /$ rh 19.7970

ISSN : $1777-5329$

Éditeur

La Société de 1848

\section{Édition imprimée}

Date de publication : 1 décembre 2021

Pagination : 225-227

ISSN : $1265-1354$

\section{Référence électronique}

François Jarrige, "Samuel BAMFORD, La Vie d'un radical anglais au temps de Peterloo ", Revue d'histoire du XIXe siècle [En ligne], 63 | 2021, mis en ligne le 01 janvier 2022, consulté le 21 février 2022. URL : http://journals.openedition.org/rh19/7970 ; DOI : https://doi.org/10.4000/rh19.7970

Ce document a été généré automatiquement le 21 février 2022.

Tous droits réservés 


\section{Samuel BAMFORD, La Vie d'un radical anglais au temps de Peterloo}

Traduction par Laurent Bury, édité par Fabrice Bensimon, avec le concours de Robert Poole, Paris, Éditions sociales, 2019

\section{François Jarrige}

\section{RÉFÉRENCE}

Samuel BAMFORD, La Vie d'un radical anglais au temps de Peterloo, traduction par Laurent Bury, édité par Fabrice Bensimon, avec le concours de Robert Poole, Paris, Éditions sociales, 2019, 400 p., 25 euros.

1 Paru à compte d'auteur entre 1839 et 1842 dans le Lancashire, la Vie d'un radical (Passages in the Life of a Radical) de Samuel Bamford constitue l'une des plus célèbres des nombreuses autobiographies ouvrières publiées en Grande-Bretagne au XIX ${ }^{e}$ siècle. De ce texte devenu un classique plusieurs fois réédité outre-Manche, l'historien Edward P. Thompson jugeait même qu'il s'agissait d'une " lecture essentielle pour tout Anglais ${ }^{1}$ ".

2 Né en 1788, Samuel Bamford fut en effet un acteur de premier plan du radicalisme anglais et l'un des organisateurs du meeting monstre de Saint Peter à Manchester, le 16 août 1819, qui se conclut par un massacre devenu fondateur de la culture politique britannique (le massacre de Peterloo). Bamford lui-même était tisserand à domicile, travaillant la soie après avoir exercé divers métiers dans la région de Manchester. Il décrit en détail les péripéties qui traversent les années 1816-1821 marquées par de vives tensions socio-politiques après la fin des guerres napoléoniennes et les débuts de l'essor industriel qui trouve dans la région l'une de ses terres d'élection.

Condamné à un an de prison pour sa participation à l'organisation du meeting de Manchester en 1819, il décrit en détail les conditions de sa captivité et celle de ses compagnons. Il en sort en 1821 et reprend alors ses activités de tisserand à domicile tout en se consacrant à l'écriture et en s'éloignant de plus en plus du radicalisme qui connaît une crise dans les années 1820 avant de renaître après 1838 avec l'essor du 
mouvement chartiste. Mais, alors âgé de 50 ans, Bamford ne se reconnaît plus dans la nouvelle génération de radicaux, il déteste la violence des discours et rejette la figure de Feargus O'Connor, et ceux qu'il décrit comme des «hommes violents et sans principes » qui finiront usés par leur "propre folie» (p. 392). C'est dans ce contexte qu'il décide de raconter sa vie et ses expériences passées, pour éclairer le présent, et fournir à la nouvelle génération radicale un pan de son histoire et un guide pour l'action. « Vingt-cinq années d'expérience âpre et brutale - depuis 1815 - autorisent un homme à avoir sa propre opinion et à l'exprimer ", observe-t-il (p. 111).

4 Les mémoires de Bamford offrent ainsi l'un des récits les plus saisissants de l'événement de Peterloo, de sa préparation aux massacres, et leurs suites. Sa plume alerte plonge le lecteur dans le contexte de l'agitation radicale du début du xix siècle, on le suit dans les tavernes, les prisons et sur les routes, à la rencontre de figures célèbres comme inconnues. On lit ainsi de savoureuses descriptions comme lorsque Bamford réunit en janvier 1819 une dizaine de « rustiques gaillards » « munis de solides gourdins, de poids et de longueurs variables » afin d'assurer la protection et la sécurité de l'orateur radical Henry Hunt tout juste arrivé à Manchester (p. 151).

5 Il s'agit néanmoins d'un récit de l'âge mûr, publié plus de 20 ans après les faits qu'il raconte, et qui reconstruit largement les circonstances de l'évènement. L'ouvrage de Bamford paraît en effet alors que l'auteur s'est éloigné du radicalisme de sa jeunesse. Il n'en propose pas moins un texte important sur la société britannique du début du XIX ${ }^{e}$ siècle, autour de l'évènement fondamental qu'est le massacre de Peterloo en 1819. C'est aussi un texte qui reflète les originalités de ce monde des artisans tisserands érudits, alphabétisés, qui n'hésitent pas à prendre la plume pour exprimer leur vision du monde, offrant une source précieuse sur la culture et le rapport à la lecture et à l'écriture des milieux populaires anglais, comme l'atteste par exemple la question des pétitions qui revient fréquemment dans le texte comme une liberté fondamentale.

6 L'auteur est à la recherche de respectabilité, il veut être lu par les puissants, il s'efforce de dialoguer avec les intellectuels et les écrivains de son temps, monde auquel il aspire à appartenir. Il dit en revanche peu de choses du travail du tisserand à proprement parler, de l'expérience ouvrière concrète, il entend d'abord s'agréger au milieu littéraire. Contemporain des débuts de l'industrialisation du pays, il observe que l'Angleterre s'est « engagée dans une lutte avide pour la richesse» (p. 395), mais il ne partage pas les réponses socialistes qui commencent à voir le jour autour de 1840, dans lesquels il ne perçoit qu' « agitation » ou « déclamations tonitruantes », « celles dont on rebat aujourd'hui les oreilles du peuple, et qui reviennent à offrir beaucoup d'énergie au vent » observe-t-il de façon méprisante (p. 394). Pour lui, seule une sage modération couplée aux droits politiques et au patriotisme peut offrir des ressources à la Nation. L'auteur est fier d'échanger avec des personnalités de son temps; il scrute de près la réception de son livre, témoignant souvent d'une forme d'égocentrisme et de quête de reconnaissance qui modèle largement son écriture et sa vision des évènements.

7 Si l'ouvrage est devenu un classique outre-Manche, il demeure peu connu ici et n'avait jamais été traduit en français. Cet oubli est désormais réparé grâce à l'initiative de l'historien Fabrice Bensimon qui a édité ce texte, rédigé une introduction qui éclaire ses conditions d'écriture et de publication, ainsi qu'un appareil critique et des illustrations très utiles pour s'orienter dans un texte dont de nombreuses références nous sont devenues obscures aujourd'hui, notamment pour un lecteur français peu familier de l'Angleterre du début du XIX ${ }^{e}$ siècle $^{2}$. La traduction réalisée par Laurent Bury est par 
ailleurs réussie, en dépit des problèmes et difficultés posés par le dialecte local impossible à rendre en français. Il faut noter aussi que cet ouvrage paru en 2019 participe d'un ensemble d'évènements et d'initiatives lancées à l'occasion du bicentenaire de Peterloo, qui demeure un évènement central et vivement débattu en Grande-Bretagne, même s'il a peu intéressé de ce côté de la Manche³

\section{NOTES}

1. Edward P. Thompson, La Formation de la classe ouvrière anglaise, Traduit de l'anglais par G. Dauvé, M. Golaszewski et M.-N. Thibault, Paris, Éditions du Seuil, coll. "Points », 2012 [1963], p. 1136.

2. Fabrice Bensimon avait précédemment édité d'autres récits autobiographiques de travailleurs anglais, notamment ceux de trois ouvriers, respectivement apprêteur de cuir, compositeurtypographe et orfèvre, présents à Paris dans la première moitié du XIX ${ }^{\mathrm{e}}$ siècle : Fabrice Bensimon (éd.), Les Sentiers de l'ouvrier. Le Paris des artisans britanniques (autobiographies, 1815-1850). Textes de John Colin, Charles Manby Smith et William Duthie, traduits de l'anglais par Sabine Reungoat, Paris, Éditions de la Sorbonne, 2017.

3. Fabrice Bensimon, "Le bicentenaire de Peterloo, entre mémoire et histoire ", Revue d'histoire du XIX ${ }^{e}$ siècle, vol. 59, no. 2, 2019, p. 145-147. 